\title{
Perceived challenges in delivering comprehensive care for patients following stroke: a qualitative study of stroke care providers in Guangdong Province, China
}

\begin{abstract}
Word count: 188)
Purpose: To understand the challenges in delivering comprehensive care for patients recovering from stroke.

Methods: A qualitative study was conducted in two tertiary hospitals with different socio-economic characteristics in Guangdong Province, Southern China. Interviews were conducted with 16 stroke care providers including doctors, nurses, rehabilitation therapists and care workers. The interviews were audiotaped, transcribed and translated from Mandarin Chinese to English. Thematic analysis was used to draw out descriptive and analytical themes relating to care providers' experiences of existing routine stroke care services and the perceptions of challenges in delivering comprehensive stroke care.
\end{abstract}

Results: The interviews with stroke care providers highlighted three key themes that hinder the capacity of the two hospitals to deliver comprehensive stroke care. First, expertise and knowledge regarding stroke and stroke care is lacking among both providers and patients; second, stroke care systems are not fully integrated, with inadequate coordination of the stroke team and inconsistency in care following discharge of stroke patients; third, stroke patients have insufficient social support.

Conclusion: While comprehensive stroke care has become a priority in China, our study highlights some important gaps in the current provision of stroke care.

Keywords: stroke; comprehensive care; thematic analysis; China

* Main text word count: 5709 


\section{Introduction}

As the world's population ages, the absolute number of people with stroke around the world has risen significantly between 1990 to 2013 [1]. This number is projected to further increase by $20.5 \%$ in 2030 [2], with stroke-related burden (quantified by disability-adjusted life years, DALYs) particularly on the rise in low- and middleincome countries (LMICs) [3]. In China, stroke was the leading cause of death in 2017 [4] and the largest single cause of disability in adulthood [5], posing a significant burden on the state economy, society and the healthcare system [6]. The health systems capacity and readiness to deliver comprehensive stroke care services in China thus requires more attention.

Comprehensive health care for stroke survivors should involve the full range of health services both during hospitalization and post-discharge $[7,8]$, and the integration of various disciplines (doctors, nurses, therapists, neuropsychologists, dieticians and social workers [9]) in order to provide continuity of care [10-12]. Multi-disciplinary stroke units are regarded as an ideal way to promote stroke recovery with confirmed benefits [13] and coordination of services among the various professionals within and beyond the health sector is vital to ensure adequate post-stroke care and rehabilitation [14]. However, in many health systems, coordination of services during hospitalization is reported to be fragmented, and care following discharge is usually disconnected from hospital care [15]. In China, the National Stroke Prevention Committee has been exploring the construction of stroke units (SU) since 2012, and China's first SU was officially launched in May 2015 [6]. At present, SUs in China are distributed in both tertiary (high-level) and primary care hospitals [16]. Further, nursing professionals have begun to carry out transitional nursing practice for stroke survivors following their discharge from hospital $[17,18]$, which aims to provide patients with holistic care [19]. 
Stroke diagnosis, treatment and rehabilitation are in the process of being gradually standardized in China, guided and regulated by the Stroke Prevention Engineering Committee of National Health Commission [20]. Once an acute stroke occurs, either the patient visits a hospital or calls for emergency treatment, the standardized procedures for diagnosis and treatment will be adopted by the nearest hospital qualified for stroke treatment. When patients are clearly diagnosed with stroke, they would be treated with thrombolysis or thrombectomy according to the guidelines and then admitted to hospital for further treatment. Stroke rehabilitation pathways are determined either by patients' attending doctors who are in charge of their treatment or through further rehabilitation therapists if patients were referred to them. More recently, the Chinese Stroke Association (CSA) has published a series of guidelines including one on stroke rehabilitation management [21], with a view to establishing more evidence-based early and long-term rehabilitation practices.

Despite these efforts, stroke is still not included in the standardized management of chronic diseases regulated by the Chinese National Health Commission, which includes, for example, hypertension and diabetes [22]. Little is known about the implementation of comprehensive stroke care delivery and multidisciplinary cooperation in Chinese hospital settings. Between 2016 and 2018, together with UK collaborative partners, we conducted a multi-disciplinary project in Guangdong Province to explore the current scope of care for older stroke patients in China as well as the options for integration of health and social care systems for their rehabilitation following a stroke event. We examined the existing services, interviewed health and social care providers, as well as older adults who had suffered from or were currently affected by a chronic illness. Additionally, we conducted a hospital-based study to examine stroke care trajectories. Within this study, we elicited patients and providers' 
perceptions and experiences of stroke care delivery from hospital admission to postdischarge. This paper draws on the interviews conducted with stroke care providers to explore the following questions: 1) what is the scope of existing care services for poststroke patients? and 2) what are the challenges in delivering comprehensive care for stroke patients?

\section{Methodology}

\section{Study design and setting}

We conducted a cross-sectional qualitative study in two tertiary hospitals, located in two cities of Guangdong Province, to explore stroke care providers’ views and experiences of delivering stroke care. The two cities have different socio-economic characteristics. Hospital 1 (hereafter referred to as H1) is located in Guangzhou, the largest city in South China and well-resourced with medical facilities as well as health care professionals [23]. Hospital 2 (hereafter referred to as H2) is located in Qingyuan city which is less well economically developed with fewer healthcare facilities and professionals compared with Guangzhou [24].

H1 and H2 have a lot in common with Chinese tertiary hospitals (see Table 1: Profile of the two hospitals at the time of investigation). Both hospitals have a neurology department; both contain 3 different wards, including a distinct stroke ward. Both hospitals have additional rehabilitation departments that aim to integrate stroke treatment and rehabilitation with designated rehabilitation doctors mainly responsible for stroke patients. Decision-making procedures regarding treatment are similar in both hospitals; all doctors strictly follow their hospitals' standardised guidelines in the acute phase of treatment; in the recovery period, patients may also be provided with treatment combining traditional Chinese medicine and Western medicine. 
It is noteworthy that stroke units and stroke-specific teams are nominally present in both hospitals, and that stroke team members do not all belong to the same ward or department. Further, in both hospitals, the stroke team members seldom participate in ward rounds together, and they mainly cooperate with each other through consultation. In other words, except ward-based doctors and nurses, other stroke-related specialists including speech-, dysphagia-, rehabilitation therapists and others only appear in the stroke ward when there is a consultation call. Besides, if the patients have psychological needs, they are referred to the psychological clinic for treatment on their own.

$<$ Table 1: Profile of the two hospitals at the time of investigation> (newly added)

\section{Study population and sampling}

The stroke care providers referred to in this study are professional staff members who deliver direct care to persons with stroke during hospitalization. Purposive sampling was used to select 16 (8 per hospital) stroke care providers, reflecting different professional roles and levels of authority, and including doctors, nurses, therapists and care workers. The selection criteria for inclusion of providers were that they were first, engaged in stroke treatment, rehabilitation, nursing or caregiving with qualifications and practising for at least 2 years; and second, willing to share their perspectives and experiences. Potential participants were identified via the head nurse of the department, who acted as a gatekeeper. Those who responded positively were then contacted to confirm their willingness to participate before each interview.

\section{Data collection}

We conducted semi-structured, face-to-face interviews in this study. The qualitative study protocol and topic guides were developed in consultation with the UK-based 
investigators (BM, KK and GYZ). Data collection was carried out by four Chinese researchers (1 nursing professor-LHW, 1 social researcher-NG, and 2 research assistants-MJC and CCW) and consisted of four stages: 1) Developing the interview guide (KK, BM, GYZ, LHW and CCW); 2) Organizing the qualitative research method training workshop (LHW, NG, MJC and CCW); 3) Piloting interviews in H1, one of the sample hospitals (LHW, NG, MJC and CCW); and 4) Conducting and recording the interviews (LHW, MJC and CCW) using a refined interview guide (See Table 2). Interviews were all conducted in Mandarin Chinese and varied considerably in length, ranging between 23 to 73 minutes. The topic guides covered the following areas of inquiry: 1) providers' experiences of delivering stroke care; 2) their views on the key care needs at different stages of stroke; and 3) their perceptions of difficulties in providing comprehensive stroke care. Interviews were conducted in a step-by-step manner, and all participants’ perceived challenges in delivering stroke care based on all their previous experiences were the focus of this paper.

All interviews were arranged at venues that were convenient for the participants, usually in a quiet room at their place of work in the two hospitals. All the audiorecorded interviews were transcribed verbatim in Mandarin [25], and then thoroughly checked for inconsistencies against the recordings by the lead author.

<Table 2: Semi-structured interview guide>

\section{Ethical considerations}

This study was approved by Queen Margaret University Research Ethics Committee, and the ethics committee of the Qingyuan People’s Hospital. Informed written consent was obtained before each interview. The interviews were conducted in quiet spaces where only the interviewer and the participant were present, in order to avoid 
interruptions and ensure confidentiality [26]. All participants were informed of the purpose of the study and were given a participant information sheet in conjunction with the consent form. Consent forms specified how the data would be handled and stored, and how confidentiality and anonymity of the participant would be guaranteed. Participants were informed that they could withdraw from the study at any time, without giving a reason. All the records as well as transcripts and analysis from the interviews were saved in encrypted files with passwords only available to the research team.

\section{Data analysis}

Transcripts were fully anonymised with names and other identifying markers omitted from all data. Thematic analysis was used to draw out descriptive and analytical themes [27]. The analysis process was led by the first author with assistance of the China-based research team (LHW, MYW, MJS, MJC and CCW) using the original Chinese transcripts and with guidance and inputs from the UK-based researchers (BM, KK and GYZ). We followed four steps: 1) Reading and re-reading all transcriptions and familiarizing ourselves with the data, then identifying meaningful statements and phrases that reflected a theme and marking these as meaning units (MYW, MJS, MJC and CCW), which could serve as the basis of an initial coding system [28]. 2) Labelling and coding of individual meaning units [25]. Four meetings were conducted, including 2 face-to-face discussions and 2 video meetings, to consider and agree on codes and a unified coding system. Independent coding of the transcripts was then conducted by MYW, MJS, MJC and CCW. 3) Translating all coded materials into English (MJS and CCW). After the Chinese and UK researchers had reviewed the coded text, another 3 online discussions involving the whole team were held to further elaborate the key themes. 4) Clarifying and defining final themes between the China-UK research team including the interviewers was undertaken through group discussion [27]. 


\section{Results}

\section{Participant profiles}

All 16 stroke care providers approached agreed to participate in this study and completed the interviews. Participants represented varied professional disciplines from the two hospitals respectively, including neurological doctors, nurses and care workers from stroke wards, and rehabilitation doctors, nurses and physiotherapists from rehabilitation wards. The mean years of practice in providing stroke care services was 10.31 years; demographic data of the participants are presented in Table 3.

<Table 3: Demographic information of participants >

\section{Perceived challenges in delivering comprehensive stroke care}

There were three overarching themes that reflect the care providers' perceptions of the current management of stroke patients and challenges in delivering comprehensive care. These are: 1) inadequate stroke knowledge; 2) partially integrated care systems; 3) insufficient social support. Specific content with details and corresponding meaning units (MU) are shown in Table 4.

$<$ Table 4: Summary of key themes, initial coding words and corresponding meaning units> (newly added)

\section{Inadequate Stroke Knowledge}

Stroke knowledge refers to both the care providers' self-assessment of their capacities in stroke care (expertise) as well as the providers' perceptions of the patients' knowledge about stroke (literacy). In this study, providers’ lack of adequate expertise and patients’ limited stroke literacy were commonly perceived and reported. 


\subsection{Providers' insufficient stroke expertise}

When asked about health care professionals' perceptions of comprehensive stroke care, the majority of them emphasized that the early diagnosis by primary care physicians and long-term rehabilitation after discharge are as important as the timely treatment in highlevel hospitals. However, primary medical care in China still needs to be developed, as stated by our participant:

There are some grassroots doctors (primary care physicians or doctors in basic medical units) who do not have scientific understanding of the disease. They invited me to give lectures in their hospitals to help raise their stroke expertise. So, we are all committed to doing this work and I believe we are all trying our best to make full use of the time after stroke onset in order to provide the prompt and crucial treatment. [MU-1. Neurological doctor, male, H2, ID10]

Our participants are all from tertiary hospitals, the highest-level hospitals in China, when asked about their capacity to manage stroke patients, there were some discrepancies in knowledge across the professions. Doctors were relatively confident in their treatment ability since they all strictly followed the standard clinical pathways, while there was a nurse expressed her further needs to improve specific stroke knowledge and professional skills:

The current caring methods we used mostly either come from our predecessors or textbooks, which are sometimes out of date, and we do not know much about the latest development about stroke care. Nevertheless, our department will introduce some new theories or nursing interventions, and I have tried to search some literature to know more, but sometimes I just cannot fully understand the latest technology. [MU-2. Neurological nurse, female, H1, ID6]

Participants from both hospitals stated that it was important to pay close attention to patients' feelings and mental health needs; however, therapists in this study 
mainly focused on physical function rehabilitation, and other aspects such as psychological health received little attention. According to the doctors and nurses in the stroke ward, psychological assessment had not been included in the routine stroke treatment though some were trying to put more personal efforts on patients' well-being. When stroke patients were found to have obvious psychological abnormalities, their responsible doctor or nurse would refer them to the psychotherapist for further evaluation and treatment:

To be honest, we really care less about patients’ psychological problems than physical problems. As for me, I always want to learn how to help patients who are reluctant to accept illness (stroke) and help them quickly solve their psychological problems, but I just have no idea where and how to learn on my own in my spare time. It would be better if there are some online training courses related to psychological care. [MU-3. Neurological nurse, female, H2, ID13]

We may not be able to accurately assess patients' mental state, but we pay attention to their behavioural disorders during ward rounds. If necessary, we will ask psychotherapist to evaluate our patients. But this is not our primary concern. [MU-4. Neurological doctor, male, H1, ID1]

There is a shortage of psychotherapists in our department, but we physiotherapists also know a little bit of psychological knowledge. So, in my case, if patients have some psychological disorder, I will communicate with them first and try to enlighten them; if that doesn't work, I will then transfer them to the psychotherapist. [MU-5. Rehabilitation therapist, male, H2, ID12]

\subsection{Patients' limited stroke literacy}

Most of the care providers repeatedly reported that patients lack awareness of stroke onset, for example, patients' delayed presentation at the hospital because they did not 
know about stroke, thereby missing the optimal treatment time. Providers also reported that many patients and their relatives had limited stroke prevention knowledge. It is worth noting that several doctors from both hospitals specifically pointed out that 'many Chinese elderly people are convinced of Traditional Chinese Medicine (TCM) and often take Chinese medicine without any doctor's advice, especially their extensive use of Angong Niuhuang Pill (ANP)', and also expressed their concerns about the side effects of ANP , as the safety and effectiveness of ANP have not been verified in academia.

From my experience, a great number of the elderly already had symptoms of hemiplegia, but they did not recognise these and did not tell their children, they just chose to eat the Angong Niuhuang Pills by themselves then just stayed at home with the belief that they could feel better as the time passed by. What a pity! [MU-6. Neurological doctor, male, H2, ID10]

Patients’ lack of knowledge about stroke was perceived by some participants to have an influence on their expectations of treatment. A neurological nurse from $\mathrm{H} 2$ (ID6) reported that some patients had insufficient knowledge of stroke treatment and expected to fully recover. Other patients were perceived to not fully understand the necessity of rehabilitation and were deemed less likely to comply with rehabilitation activities.

I notice that many patients do not know the importance of rehabilitation and consider medications to be more important. From the conversations I had with the patients I was responsible for before, I found that they always think that stroke as a kind of disease should rely on medication, and there is no need to do rehabilitation by themselves. It's a bit sad to hear these words, but this kind of cognitive prejudice is very common, because most elderly people have not been exposed to rehabilitation knowledge, and that's why we need to continuously provide health 
education to patients and their families. [MU-7. Neurological nurse, female, H1, ID5]

\section{Partially Integrated Care Systems}

In line with comprehensive stroke care, a holistic care system centered on stroke patients should contain both hospital-based elements of care and as well as follow-up care after discharge. In this study, both inadequate coordination of the multidisciplinary stroke team and inconsistency in care following discharge by stroke wards emerged as perceived challenges in achieving the fully integrated hospital-based care system.

\subsection{Inadequate coordination of multidisciplinary stroke team}

Decision-making about treatment appeared to be predominantly influenced by doctors’ professional opinions according to established routines. When asked about the implementation of teamwork in stroke units or multidisciplinary cooperation, all health professionals responded that they mainly use the consultation system to achieve cooperation among different disciplines. Specifically, consultation systems of H1 and H2 are basically the same as Chinese tertiary hospitals, including emergency consultation and ordinary consultation. When stroke patients need medical support from other departments or disciplines, the responsible doctor will send them an application for consultation through the medical system, then doctors or therapists who receive those consultations will come to the stroke ward within the prescribed time, depending on hospital requirements.

We usually ask the responsible department for consultation if we have some specialist problems. It is a common rule in our hospital that all consultations should be done within 48 hours, and I think our department implements it quite well. To be specific, after the consultation, the corresponding doctor will write down his own 
opinions and prescriptions. If we agree with that, we will follow. However, for patients with complex conditions, our director will organize doctors and nurses from different disciplines to conduct face-to-face discussions. [MU-8. Neurological doctor, male, H2, ID10]

Nurses from both hospitals also stated that there were separate nursing consultations between nurse professionals across different departments. However, when asked about the actual attendance at multidisciplinary meetings or rounds, doctors and therapists generally responded they would be asked to participate, ranging from once a week or once a month; while the participation of nurses is relatively inadequate, and usually only the nurse leaders could attend:

In our department, we attach great importance to the communication and cooperation between our nurses and doctors as well as therapists. Besides, two nurse leaders in our ward and I (head nurse) will be required to participate the rounds with our director and all doctors regularly, which can strengthen our understanding of some specific or new treatment for patients under different conditions. [MU-9. Neurological nurse, female, H1, ID5]

We nurses are most familiar with our patients' physical condition because we spend most our time with them, and usually we first discover the changes of patients' condition then report it to the responsible doctor. Our department (should) also have multidisciplinary cooperation, we have the large-scale meetings every now and then attended by many people, mainly doctors and also our head nurse. I have never been asked to attend, and our head nurse will tell us some key contents after her participation. [MU-10. Neurological nurse, female, H2, ID13]

\subsection{Inconsistent follow-up care after discharge}


Follow-up care after discharge was emphasized in the study, however, there were no standard routines to support patients' post-discharge care in all of the participants' reported experiences. Discharge notifications were commonly reported to be doctor-led decisions, while afterward, the doctors' responses to whether there was a post-discharge plan were mostly negative in both hospitals:

When our patients are in stable condition, we will inform them to be discharged, and at the same time, we will make a discharge summary for each patient as a medical record. There is no requirement that we should provide patients with such a discharge plan, and I usually give my patients some recovery suggestions. Because different hospitals have different requirements. No matter which hospital or rehabilitation center our patients go to after discharge, the doctors there will certainly re-evaluate them and then make their treatment or rehabilitation plan following their standards. [MU-11. Neurological doctor, male, H1, ID2]

Both hospitals have regular telephone follow-up after discharge, which was initially carried out by both responsible nurses and doctors within the wards. With the development of the chronic disease management team of $\mathrm{H} 1$ and the establishment of a dedicated follow-up department in H2, these two teams are responsible for the followup of all discharged patients through each department/ward referring their patients who need long-term follow-up to these two separate teams respectively. Therefore, the follow-ups of both hospitals have gradually developed into a process jointly performed by the stroke ward and the corresponding team, while the ward medical staff gradually reduced their participation.

At first, we have a strict follow-up timeline after patients' discharge by our nurses who are responsible for them, at about a week, the third and sixth month. While to date, we do not need to do so since the establishment of the 'Follow-up 
Department' last year, and they will continue to follow up and record the patients after our follow-up, but I don't know how long their specific follow-up will take, which might be based on the patients' recovery. [MU-12. Neurological nurse, female, H2, ID14]

\section{Insufficient Social Support}

In addition to hospital-based stroke care, the social support including financial protection, family care and available medical resources in community settings were perceived to vary greatly.

\subsection{Incomplete coverage of medical reimbursement}

In China, the proportion of patients' medical expenses reimbursement is complex and varies depending on whether the patient's household registration is rural or urban, hospital or outpatient costs, medicine or surgical expenses as well as the types of disease they suffer and the types of health insurance they have. It is noteworthy in this study that, the limited coverage of the current health insurance reimbursement scheme for stroke was stated as a key barrier to receiving good quality care. Participants expressed ambivalence about China’s health insurance system:

Most of our treatment costs cannot be reimbursed, which may limit patients' recovery. ... Our rehabilitation treatment technology is in line with foreign countries, but the insurance system and policy do not match. Compared with other countries, our policy is lagging behind, which is a big obstacle to the development of our rehabilitation. Some patients are not in good financial positions and we will encourage them to go to community hospitals for rehabilitation. After all, the proportion of reimbursements for there will be much higher than our hospital (tertiary hospital). [MU-13. Rehabilitation doctor, male, H1, ID3] 
In addition, from the perspective of national macro-control, the government will refer to the overall database of Chinese patients to control the average medical expenses according to the different types and severity of the diseases, and then take measures to control or limit the medical expenses of certain diseases based on this. Accordingly, the health insurance system could affect hospital revenues by restricting the cost of hospitalization and the length of stay, thereby affecting the income of individuals. On this basis, rehabilitation doctors reiterated the political pressures caused by hospital rules relating to the insurance policy:

Because national policy needs to control the proportion of patients' medical reimbursement, then at the hospital level, the patient's length of stay and cost of hospitalization need to be controlled. But in reality, it is often difficult to control either the cost or the length. If the medical insurance reimbursement amount of our department patients exceeds, then the excess part will be paid by our department thus the overall performance bonus of our department will decrease. [MU-14. Rehabilitation doctor, male, H2, ID11]

\subsection{Lack of family care and community support}

The onset of stroke was seen to place a heavy burden of care on the families, as stated generally among our participants. The lack of family care and support emerged as a recurrent theme:

Patients in intensive care units usually have serious condition. They might not improve by being treated for only one or two months... these patients tend to have obvious sequelae, often not able to take care of themselves anymore...they cannot always live in the hospital, which will add the care burden to family members. But most family members complain that they have no capacity to take care of the 
patient, then care comprehensiveness therefore cannot be guaranteed. [MU-15. Neurological doctor, male, H1, ID1]

When asked about perception of care after discharge and understanding of available medical resources of their own patients, doctors from both hospitals highlighted the significance of community health support and emphasized the need to develop primary care in the community:

At present, our country's community-based care is still not well developed, and even not existent in some rural areas. Now our hospital is in the process of conducting and promoting home rehabilitation, and we are thinking about how to carry it out. After all, home-based rehabilitation is more difficult to implement despite its benefits. [MU-16. Rehabilitation doctor, male, H1, ID3]

It is impossible for all patients to get crowded in tertiary hospitals for rehabilitation. Therefore, state or local government should increase the construction and development of community hospitals or clinics, for example, there should be some medical centers in each street. As far as I know, community care for stroke is currently available and processing in many big cities, but it is still very scarce and insufficient in small cities or rural areas. So, I think that primary health care in the community still needs strong government support to develop better; after all, the development of community-based care will greatly enhance the convenience of treatment and rehabilitation of stroke patients, thereby improving comprehensive stroke care. [MU-17. Neurological doctor, male, H2, ID9]

\section{Discussion}

The study identified three key themes arising from hospital providers' perceptions of the experiences and challenges of delivering comprehensive stroke care in these two Chinese hospitals, namely, knowledge of stroke, limitations to the integration of 
services and inadequate social support.

The number of stroke patients will continue to rise with an ageing population [29], thus timely arrival to hospital and prompt treatment are crucial. However, delays in accessing emergency services as reported in this study were seen to relate not only to patients' limited stroke knowledge but some primary care providers' insufficient expertise, a finding consistent with previous studies conducted in China [30,31]. Older people in China who have had a stroke generally believe in Traditional Chinese Medicine (TCM), which has been proposed as a potential candidate for stroke treatment [32]. However, public awareness of stroke symptoms remains insufficient, and it is worrying that some stroke patients excessively use TCM such as Angong Niuhuang Pills (ANP) without a physician' prescription. To date, the effectiveness and safety of ANP has not been confirmed by clinical randomized controlled trials [33], thus the need for improving knowledge of stroke among older people and stroke patients should be highlighted and prioritised. Fortunately, stroke health education programs conducted in hospitals are gradually increasing in China [34], and Chinese Stroke Association [35] also plays a key role through its conferences and online platforms. In addition, as the first point of contact for citizens, community physicians have the potential to act as key knowledge disseminators [36]. It is expected that more training programs will be available for the primary care physicians before they can carry out some high-quality publicity and education activities in the community setting, thereby helping raise population awareness of stroke symptoms and minimize the adverse consequences of stroke.

Coordinated multidisciplinary treatment and rehabilitation are the gold standard of quality stroke services, and should exist through the whole care process. In this study, 
although all participants were selected from either the stroke ward or the rehabilitation department which all belong to the stroke unit (SU) in the two hospitals, they seldom mentioned the SU or multidisciplinary cooperation. Instead, the consultation system was reported to be the sole form of cooperation among various departments and played an important role in the context of the Chinese medical environment. As evidenced from previous research [37], in order to improve stroke care, the integration of organizational resources should always be emphasised despite clinical-administrative processes. While the multidisciplinary stroke ward rounds were infrequently referred to in our participants' reports (see Table 1), it is also noteworthy that nurses expect for chief nurses were rarely involved in multidisciplinary rounds or meetings. An increasing number of studies suggest that nursing specialists could provide quality stroke care $[38,39]$, thus it is necessary to maximize the coordination role of nurses in the multidisciplinary team, so as to provide better comprehensive care to stroke survivors.

The development of medical technology has reduced stroke mortality rates, resulting in stroke survivors living with disabilities in their advancing years. As a result, post-hospital long-term care is needed to optimize recovery, minimize disability and maintain independence for as long as possible. Long-term care (LTC) has been defined by the WHO [40] as the system of activities undertaken by professionals and/or informal caregivers to ensure that a person who is not fully capable of self-care can maintain the highest possible quality of life. This definition is similar to that of the National Institute on Aging (NIA) [41] who state that LTC involves a variety of services to help people live as independently and safely as possible. As recommended in the NIA guide, stroke survivors' long-term care should combine both formal care delivered by the hospital professionals and informal care provided by their families or relatives. 
However, from the interviews with hospital care providers in this study, the formal part of LTC was mainly carried out separately by the responsible ward-based doctors and nurses and with both groups having different treatment and care foci. However, even this kind of practice was only evident in the specialized follow-up facility, but even there, an integrated approach was not evident. As for the informal part of LTC, home care has been proven to be associated with improved quality of life and reduced dependency among stroke patients in China [42], while the lack of family support was highlighted by our participants as something that was evident in the majority of cases, despite some patients having quality family care. Therefore, it is clear that for the ambition of more integrated hospital and post-hospital (LTC) care services be realised, there is a need to develop more standardized systems and processes that would optimize the integration of care for patients with stroke.

A comprehensive and integrated stroke service needs to include a support system to ensure patients continue to receive optimal care during the whole recovery period. However, a key consideration here is the cost burden of stroke, since there is increasing evidence of families of stroke patients facing considerable economic hardship after stroke $[43,44]$. In our study, financial constraints were reported to be the barriers to implementing optimal stroke care, similar to some LMICs [45]. Fortunately, China's medical insurance policy continues to improve, and almost all the Chinese population (95\%) have varied health insurance since 2012 [46]. However, the cost of stroke rehabilitation still cannot be fully reimbursed by the insurance system [16], so the financial burden on stroke survivors and their families remains high. The influence of the current medical insurance system of China also has an influence on clinical decision-making as well as impacting the quality of care that patients and families receive. This situation can also result in a sense of powerlessness and a lack of 
motivation to provide effective care.

This study has several limitations. First, we only included two general hospitals in Guangdong Province thus it may lack representativeness of site selection and generalizability of our findings. Second, we did not include all stroke-related professional disciplines when selecting stroke care providers, such as not incorporating for example the role of psychological therapist, dietitian, and speech therapist. Although the personnel selection was based on the actual situation of the hospitals, it still lacked comprehensiveness. Another limitation is the lack of specific information about hospital human resources and departmental performance, because it was challenging to get access to these dynamic and confidential data. Despite these limitations, our study highlights key issues for consideration in the development of comprehensive and integrated stroke services in China and learning from the findings of this research may have more wide-ranging applicability.

\section{Conclusion}

This study provides a macroscopic perspective of health care providers' experiences of providing stroke care services in the context of Guangdong Province, Southern China. Our interviews indicated that comprehensive stroke care in China is making progress, but there are still gaps in service provision. Our findings expose some major challenges perceived by the care providers, including a lack of professional expertise in stroke care, patients’ limited stroke literacy and only partially integrated care systems. These issues require a sustained government supported driver for integration in and between care services, as well as increased investment in community-based primary care systems. However, there is also much work to be done within the hospital system itself to address the insufficient stroke expertise and team coordination. In-house programs of training 
and development are needed, drawing on best evidence in comprehensive stroke care and practices. Only by improving the providers' care skills and increasing cooperation between different disciplines can they deliver better quality care. Participants in this research also highlight key issues that are underpinned by the national model of financial support. Overall, our research highlights issues to be addressed in all parts of the health and care system should the ambition of comprehensive stroke services be realised.

\section{References}

1. Feigin VL, Mensah GA, Norrving B, et al. Atlas of the Global Burden of Stroke (1990-2013): The GBD 2013 Study. Neuroepidemiology. 2015;45(3):230-6.

2. Writing Group Members, Mozaffarian D, Benjamin EJ, et al. Heart Disease and Stroke Statistics-2016 Update: A Report From the American Heart Association. Circulation. 2016 Jan 26;133(4):e38-360.

3. Feigin VL, Roth GA, Naghavi M, et al. Global burden of stroke and risk factors in 188 countries, during 1990-2013: a systematic analysis for the Global Burden of Disease Study 2013. Lancet Neurol. 2016 Aug;15(9):913-924.

4. Zhou M, Wang H, Zeng X, et al. Mortality, morbidity, and risk factors in China and its provinces, 1990-2017: a systematic analysis for the Global Burden of Disease Study 2017. Lancet. 2019 Jun 24.

5. Zhou MG, Wang HD, Zhu J, et al. Cause-specific mortality for 240 causes in China during 1990-2013: a systematic subnational analysis for the Global Burden of Disease Study 2013. Lancet. 2016 Jan 16;387(10015):251-272.

6. Wang; L, Wang; J, Peng; B, et al. Summary of Chinese stroke prevention report 2016. Chinese Journal of Cerebrovascular Diseases. 2017;14(4):217-224.

7. Assis MM, do Nascimento MA, Pereira MJ, et al. Comprehensive health care: dilemmas and challenges in nursing. Rev Bras Enferm. 2015 MarApr;68(2):304-9, 333-8.

8. Iihara K, Nishimura K, Kada A, et al. The impact of comprehensive stroke care capacity on the hospital volume of stroke interventions: a nationwide study in Japan: J-ASPECT study. J Stroke Cerebrovasc Dis. 2014 May-Jun;23(5):100118.

9. Intercollegiate Stroke Working Party. National Clinical Guideline for Stroke. 5th ed. London: Royal College of Physicians; 2016.

10. Hughes PA. Comprehensive care of adults with acute ischemic stroke. Crit Care Nurs Clin North Am. 2011 Dec;23(4):661-75.

11. Miller EL, Murray L, Richards L, et al. Comprehensive overview of nursing and interdisciplinary rehabilitation care of the stroke patient: a scientific statement from the American Heart Association. Stroke. 2010 Oct;41(10):2402-48. 
12. Goodwin N. Understanding Integrated Care. Int J Integr Care. 2016 Oct 28;16(4):6.

13. Di Carlo A, Lamassa M, Wellwood I, et al. Stroke unit care in clinical practice: an observational study in the Florence center of the European Registers of Stroke (EROS) Project. Eur J Neurol. 2011 May;18(5):686-694.

14. Rodgers H, Price C. Stroke unit care, inpatient rehabilitation and early supported discharge. Clin Med. 2017 Apr;17(2):173-177.

15. Wissel J, Olver J, Sunnerhagen KS. Navigating the Poststroke Continuum of Care. J Stroke Cerebrovasc. 2013 Jan;22(1):1-8.

16. Han YH, Liu YL, Zhang XL, et al. Chinese family caregivers of stroke survivors: Determinants of caregiving burden within the first six months. J Clin Nurs. 2017 Dec;26(23-24):4558-4566.

17. Wong FKY, Yeung SM. Effects of a 4-week transitional care programme for discharged stroke survivors in Hong Kong: a randomised controlled trial. Health Soc Care Comm. 2015 Nov;23(6):619-631.

18. Geng G, He W, Ding L, et al. Impact of transitional care for discharged elderly stroke patients in China: an application of the Integrated Behavioral Model. Top Stroke Rehabil. 2019 Dec;26(8):621-629.

19. Yeung SM, Wong FKY, Mok E. Holistic concerns of Chinese stroke survivors during hospitalization and in transition to home. J Adv Nurs. 2011 Nov;67(11):2394-2405.

20. Stroke Prevention Engineering Committee of National Health Commission. Chinese Stroke Guidelines-2015 Release: wsbnfw2016; 2016. Available from: https://sinosc.org/NewsInfo/News/NewsDetailWeb? Tid $=2065 \&$ cCode $=00140003$

21. Chinese Stroke Association Guidelines Committee for Cerebrovascular Disease Clinical Management. Chinese cerebrovascular disease clinical management guide (selected edition)—_ Stroke Rehabilitation Management. Chinese Journal of Stroke. 2019;14(8):823-831.

22. National Health And Family Planning Commission. Announcement of the national health and family planning commission on the issuance of national basic public health service standards (third edition) 2017. Available from: http:// www.nhc.gov.cn/jws/s3578/201703/d20c37e23e1f4c7db7b8e25f34473e1b.shtm $\underline{1}$

23. Municipal Statistics Bureau. Statistical Communique of National Economic and Social Development of Guangzhou City in 2018 2019. Available from: https://www.gz.gov.cn/gzstatsjg/tjsj/201904/49957c36f9f84cdc95978e3aa5caf2 d6.shtml

24. Municipal Statistics Bureau. Statistical Communique of National Economic and Social Development of Qingyuan City in 2018 2019. Available from: http://www.qystats.gov.cn/qystjj/tjgb/201903/d469640ea0634a309ddc1941da5d bb69.shtml

25. Ziebland S, McPherson A. Making sense of qualitative data analysis: an introduction with illustrations from DIPEx (personal experiences of health and illness). Med Educ. 2006 May;40(5):405-414.

26. Kaiser K. Protecting Respondent Confidentiality in Qualitative Research. Qual Health Res. 2009 Nov;19(11):1632-1641.

27. Braun V, Clarke V. Using thematic analysis in psychology. Qualitative Research in Psychology. 2006;3(2):77-101. 
28. Giorgi A. The theory, practice and evaluation of the phenomenological method as a qualitative research procedure. Journal of Phenomenological Psychology. 1997;28(2):235-260.

29. Feigin VL, Forouzanfar MH, Krishnamurthi R, et al. Global and regional burden of stroke during 1990-2010: findings from the Global Burden of Disease Study 2010. Lancet. 2014 Jan 18;383(9913):245-54.

30. Wan LH, Zhao J, Zhang XP, et al. Stroke Prevention Knowledge and Prestroke Health Behaviors Among Hypertensive Stroke Patients in Mainland China. J Cardiovasc Nurs. 2014 Mar-Apr;29(2):E1-E9.

31. Chen C, Qiao X, Kang H, et al. Community physicians' knowledge of secondary prevention after ischemic stroke: a questionnaire survey in Shanxi Province, China. BMC Med Educ. 2015 Nov 3;15:197.

32. Zhang X, Liu XT, Kang DY. Traditional Chinese Patent Medicine for Acute Ischemic Stroke: An Overview of Systematic Reviews Based on the GRADE Approach. Medicine (Baltimore). 2016 Mar;95(12):e2986.

33. Liu H, Yan Y, Pang P, et al. Angong Niuhuang Pill as adjuvant therapy for treating acute cerebral infarction and intracerebral hemorrhage: A meta-analysis of randomized controlled trials. J Ethnopharmacol. 2019 Jun 12;237:307-313.

34. Su Q, Li C, Long F, et al. Effects of a health promotion program on medication adherence to antiplatelet therapy among ischemic stroke patients in Hainan Province, China. Vascular. 2017 Jun;25(3):242-248.

35. Chinese Stroke Association. Academic Information and Activities 2019. Available from: http://www.chinastroke.net/News.aspx?cla=1\&zcla=19

36. Soulleihet V, Nicoli F, Trouve J, et al. Optimized acute stroke pathway using medical advanced regulation for stroke and repeated public awareness campaigns. Am J Emerg Med. 2014 Mar;32(3):225-232.

37. Longpre C, Dubois CA. Fostering development of nursing practices to support integrated care when implementing integrated care pathways: what levers to use? Bmc Health Serv Res. 2017 Nov 29;17.

38. Javier Catangui E, John Roberts C. The lived experiences of nurses in one hyper-acute stroke unit. Br J Nurs. 2014 Feb 13-26;23(3):143-8.

39. Jones B, Taylor K, Dorssom J. Advanced Practice Registered Nurses Provide Cost-effective, Quality Stroke Care. Stroke. 2013 Feb;44(2).

40. World Health Organization. Towards an International Consensus on Policy for Long-Term Care of the Ageing. 2000. Available from: https://www.who.int/ageing/publications/long term care/en/

41. National Institute on Aging. What Is Long-Term Care? 2017. Available from: https://www.nia.nih.gov/health/what-long-term-care

42. Chen L, Sit JW, Shen X. Quasi-experimental evaluation of a home care model for patients with stroke in China. Disabil Rehabil. 2016 Nov;38(23):2271-6.

43. Heeley E, Anderson CS, Huang Y, et al. Role of health insurance in averting economic hardship in families after acute stroke in China. Stroke. 2009 Jun;40(6):2149-56.

44. Wei JW, Heeley EL, Jan S, et al. Variations and determinants of hospital costs for acute stroke in China. PLoS One. 2010 Sep 28;5(9).

45. Baatiema L, de-Graft Aikins A, Sav A, et al. Barriers to evidence-based acute stroke care in Ghana: a qualitative study on the perspectives of stroke care professionals. BMJ Open. 2017 Apr 27;7(4):e015385.

46. Blumenthal D, Hsiao W. Lessons from the East - China's Rapidly Evolving Health Care System. New Engl J Med. 2015 Apr 2;372(14):1281-1285. 
